PROCEEDINGS OF THE

AMERICAN MATHEMATICAL SOCIETY

Volume 139, Number 8, August 2011, Pages 2793-2805

S 0002-9939(2011)10715-9

Article electronically published on February 22, 2011

\title{
THE GL-L.U.ST. CONSTANT AND ASYMMETRY OF THE KALTON-PECK TWISTED SUM IN FINITE DIMENSIONS
}

\author{
Y. GORDON, M. JUNGE, M. MEYER, AND S. REISNER \\ (Communicated by Nigel J. Kalton) \\ Dedicated to the memory of Nigel J. Kalton
}

\begin{abstract}
We prove that the Kalton-Peck twisted sum $Z_{2}^{n}$ of $n$-dimensional Hilbert spaces has a GL-l.u.st. constant of order $\log n$ and bounded GL constant. This is the first concrete example which shows different explicit orders of growth in the GL and GL-l.u.st. constants. We also discuss the asymmetry constants of $Z_{2}^{n}$.
\end{abstract}

\section{INTRODUCTION}

Local unconditional structure, or l.u.s.t., is an important notion in the study of the geometry of Banach spaces (see for instance GL1, $\overline{\mathrm{KT}}$, $[\mathrm{PW}$ ). The variant of l.u.st. that we investigate here was introduced by Gordon and Lewis GL1] and is frequently referred to as GL-l.u.st.; another, formally more restrictive, notion of l.u.st. was introduced in [FJT].

This notion was not, however, fully studied in the finite-dimensional case, where the asymptotic values of the various constants that it involves are not yet fully understood. We show in this paper that in the finite-dimensional setting of $n$ dimensional normed spaces, the GL constant and the l.u.s.t. constant can be of significantly different orders of magnitude, by considering the $2 n$-dimensional KaltonPeck twisted sum of $n$-dimensional Hilbert spaces.

Let us now recall some definitions (GL1]). A basis $B=\left(b_{i}\right)_{i \in I}$ for a Banach space $E$ is called unconditional if there is a constant $C>0$ such that for every $x \in E, x=\sum_{i \in I} \xi_{i} b_{i}$ and every choice of signs $\varepsilon_{i}= \pm 1$, with $\varepsilon_{i}=1$ for all but a finite number of $i \in I$, one has

$$
\text { \| } \sum_{i \in I} \varepsilon_{i} \xi_{i} b_{i}\|\leq C\| x \| .
$$

The smallest $C$ satisfying this is called the unconditional constant of $B$ and denoted by $\chi(B)$. The unconditional constant of $E$ is

$$
\chi(E):=\inf \{\chi(B) ; B \text { is a basis of } E\} .
$$

Received by the editors March 12, 2010 and, in revised form, July 21, 2010.

2010 Mathematics Subject Classification. Primary 46B20; Secondary 46B07.

Key words and phrases. Banach spaces, local unconditional structure, asymmetry.

The first, third and fourth authors were supported in part by the France-Israel Research Network Program in Mathematics contract \#3-4301.

The second author was supported in part by NSF grant DMS-0901457.

(C)2011 American Mathematical Society Reverts to public domain 28 years from publication 
More generally, define the local unconditional structure constant of $E$ (see GL1]), $\chi_{u}(E)$, by

$$
\chi_{u}(E):=\sup _{F \subset E}\left(\inf _{A, B, U}\|B\| \chi(U)\|A\|\right),
$$

where the sup is taken over all finite-dimensional subspaces $F$ of $E$ and the infimum over all Banach spaces $U$ and all continuous operators $A: F \rightarrow U$ and $B: U \rightarrow E$ such that $B A=I_{F}$, the identity on $F$. Clearly $\chi_{u}(E) \leq \chi(E)$. By [FJT], $\chi_{u}(E)=$ $\chi_{u}\left(E^{\prime}\right)$ and $\chi_{u}(E)$ is finite iff $E^{\prime \prime}$ is isomorphic to a complemented subspace of a Banach lattice.

Given a subset $\left\{x_{i} ; i \in I\right\}$ of $E$, we denote

$$
\varepsilon_{1}\left(\left\{x_{i} ; i \in I\right\}\right):=\sup \left\{\sum_{i \in I}\left|x^{\prime}\left(x_{i}\right)\right| ; x^{\prime} \in E^{\prime},\left\|x^{\prime}\right\| \leq 1\right\} .
$$

Let $E$ and $F$ be Banach spaces. The weakly nuclear norm of a linear operator $u: E \rightarrow F$, which has a representation $u=\sum_{i \in I} x_{i}^{\prime} \otimes y_{i}$, which converges unconditionally in $L(E, F)$, is defined by

$$
\eta(u)=\inf \varepsilon_{1}\left(\left\{x_{i}^{\prime} \otimes y_{i}, i \in I\right\}\right),
$$

where the infimum is taken over all such representations of $u$.

By Proposition 1.2. of [GL2], one has for all $u \in L(E, F)$

$$
\eta(u)=\inf \|A\| \chi(U)\|B\|,
$$

where the infimum is taken over all factorizations $u=B A$, with $A: E \rightarrow U$ compact, $B: U \rightarrow F$, and $U$ is a Banach space with an unconditional basis. Hence $\eta\left(I_{E}\right)=\chi_{u}(E)$. It is easy to show, using e.g. techniques from $\mathrm{R}$, that when $E=F$ is finite dimensional and $u=I_{E}$, one may restrict this infimum to spaces $U$ satisfying $\chi(U)=1$ and $A: E \rightarrow U$ to be an isometric embedding.

The Gordon-Lewis constant of $E$, denoted by $\operatorname{gl}(E)$, is defined to be

$$
\operatorname{gl}(E)=\inf \left\{c>0 ; \gamma_{1}(A) \leq c \Pi_{1}(A) ; F \text { a Banach space and } A: E \rightarrow F\right\} .
$$

Here

$$
\gamma_{1}(A)=\inf \left\{\|\alpha\|\|\beta\| ; \beta \alpha=i_{F} A\right\}
$$

where the infimum ranges over all Banach spaces $F$ and all $\alpha: E \rightarrow L^{1}(\mu), \beta$ : $L^{1}(\mu) \rightarrow F^{\prime \prime}, i_{F}: F \rightarrow F^{\prime \prime}$ is the canonical inclusion, and

$$
\begin{array}{r}
\Pi_{1}(A)=\inf \left\{C>0 ; \sum_{i=1}^{n}\left\|A x_{i}\right\| \leq C \sup _{ \pm}\left\|\sum_{i=1}^{n} \pm x_{i}\right\|\right. \\
\text { for every } \left.n \geq 1 \text { and } x_{1}, \ldots, x_{n} \in E\right\}
\end{array}
$$

is the classical 1-absolutely summing norm of $A$. It was proved in GL1] that $\operatorname{gl}(E) \leq$ $\chi_{u}(E)$, and it is well known that there exist infinite-dimensional separable Banach spaces $E$ such that $\mathrm{gl}(E)$ is finite and $\chi_{u}(E)$ is infinite (the first such example is presented in [JLS] $)$. In particular, it follows that there exists an increasing sequence of $2 n$-dimensional Banach spaces $E_{n}$ for which $\chi_{u}\left(E_{n}\right) \rightarrow \infty$ and $\left(\operatorname{gl}\left(E_{n}\right)\right)_{n}$ is bounded.

We prove in the sequel that, for some constant $c>0$, one has

$$
c_{n}=\sup \left\{\frac{\chi_{u}\left(E_{n}\right)}{\operatorname{gl}\left(E_{n}\right)} ; E_{n} \text { is an } n \text {-dimensional normed space }\right\} \geq c \log n .
$$


To do this, we refine the proof, given by JLS, that the Kalton-Peck space $Z_{2}$ (see $\left[\mathrm{KP}\right.$ ) has bounded gl-constant but that $\chi_{u}\left(Z_{2}\right)$ is infinite. The spaces $E_{n}$ mentioned above are the subspaces $Z_{2}^{n}$ of $Z_{2}$ spanned by first $2 n$ coordinates. Note that always $c_{n} \leq \sqrt{n}$. An interesting problem is, how big can $c_{n}$ be? For example, is $c_{n} \geq c n^{\alpha}$ for some $\alpha>0$ and $c$ an absolute positive constant? Perhaps this is true even with $\alpha=1 / 2$.

$Z_{2}$ (and $Z_{p}$ ) has unconditional finite-dimensional decomposition into 2-dimensional subspaces (2-UFDD). Many of the concrete examples of spaces with GL and without l.u.st. possess such structure (see [KT] for references). The paper [CK] presents a general treatment of spaces having uniform UFDD (that is, UFDD for which the dimensions of the blocks are uniformly bounded). It is shown there that such a (infinite-dimensional) space either has an unconditional basis or fails to have l.u.st. Observing the computations made in the works mentioned above, it is plausible that if $c_{n} \geq c n^{\alpha}$ for some $\alpha>0$, then the examples showing this would not be with uniform UFDD. In this respect we may quote a conjecture that Nigel Kalton sent us, together with other helpful suggestions, in response to a preprint version of the present paper:

Conjecture. If we restrict the definition of $c_{n}$ to $k n$-dimensional initial blocks of spaces with a $k-U F D D$, then $c_{n}$ is equivalent to $(\log n)^{k-1}$.

The concept of asymmetry of an $n$-dimensional Banach space was introduced in GG and generalized the notion of the asymmetry of a basis. This was followed up in GL1] as well. Here we study related notions of asymmetry and apply them to $Z_{2}^{n}$.

For general terminology concerning the geometry of Banach spaces we refer the reader to [LT1, LT2. Terminology concerning normed ideals of operators may be found in $[\mathrm{P}]$ and $[\mathrm{T}]$.

Finally, we wish to thank Alexander Litvak for very helpful discussions.

\section{The L.U.ST. CONSTANT OF $Z_{2}^{n}$}

Let $Z_{2}^{n}$ be the $2 n$-dimensional (real) Banach space which is the subspace of the Kalton-Peck $\mathrm{KP}$ space $Z_{2}$, spanned by the first $2 n$ coordinates. More precisely, for $a, b \in \mathbb{R}^{n}, a=\left(a_{1}, \ldots, a_{n}\right), b=\left(b_{1}, \ldots, b_{n}\right) \in \mathbb{R}^{n}$, we define

$$
\begin{aligned}
& \|(a, b)\|_{Z_{2}^{n}}=\left\|\left(a_{1}, \ldots, a_{n}, b_{1}, \ldots, b_{n}\right)\right\|_{Z_{2}^{n}}=\left\|\sum_{j=1}^{n}\left(a_{j} e_{j}+b_{j} f_{j}\right)\right\|_{Z_{2}^{n}} \\
& =\left(\sum_{j=1}^{n} b_{j}^{2}\right)^{1 / 2}+\left(\sum_{j=1}^{n}\left(a_{j}-b_{j} \log \left(\left|b_{j}\right|\left(\sum_{i=1}^{n} b_{i}^{2}\right)^{-1 / 2}\right)\right)^{2}\right)^{1 / 2} .
\end{aligned}
$$

As it is proved in $\overline{\mathrm{KP}}$, this quasi-norm on $\mathbb{R}^{2 n}$ is (uniformly in $n$ ) equivalent to a norm.

As in [JLS] we consider $Z_{2}^{n}$ as an unconditional sum

$$
Z_{2}^{n}=\sum_{j=1}^{n} \bigoplus E_{j},
$$

where $E_{j}=\left[e_{j}, f_{j}\right]$. 
It was proved in JLS that $Z_{2}$ fails to have l.u.st. They observed that if a Banach space $E$ is of the form $E=\bigoplus_{j=1}^{\infty} E_{j}$, a 1-unconditional sum of finite-dimensional subspaces, then

$$
\operatorname{gl}(E) \leq \sup _{j} \operatorname{dim}\left(E_{j}\right)
$$

and thus $g l\left(Z_{2}\right) \leq 2 c$. Hence it follows that $g l\left(Z_{2}^{n}\right) \leq 2 c$, but that the l.u.st. constants of $Z_{2}^{n}$ tend to infinity with $n$. Here we establish the order of growth of these constants.

Theorem 1. One has $g l\left(Z_{2}^{n}\right) \leq 2 c$, and the l.u.st. constant $\chi_{u}\left(Z_{2}^{n}\right)$ of $Z_{2}^{n}$ satisfies

$$
\chi_{u}\left(Z_{2}^{n}\right) \sim \log n
$$

Throughout the proof the letters $C, c, c_{1}, c_{2}, \ldots$, etc. will denote absolute constants which do not depend on $n$. The same letter $c$ (etc.) may denote different constants in different lines.

It is shown in $\left[\mathrm{KP}\right.$ that the Banach-Mazur distance $d\left(Z_{2}^{n}, \ell_{2}^{2 n}\right)$ from $Z_{2}^{n}$ to $\ell_{2}^{2 n}$ is of the order of $\log n$. Using Proposition 2 of [JLS], there exists a Banach space $Y_{n}$ with a 1 -unconditional basis $\left\{y_{j, i}, j=1, \ldots, n, \quad i=1, \ldots, k_{j}\right\}$, such that

- $Z_{2}^{n}$ is a subspace of $Y_{n}$ and, for each $1 \leq j \leq n, E_{j} \subset\left[\left\{y_{j, i}\right\}, i=1, \ldots, k_{j}\right]$.

- There exists a projection $P_{n}: Y_{n} \rightarrow Z_{2}^{n}$ such that $P_{n}\left(\left[\left\{y_{j, i}, i=1, \ldots, k_{j}\right\}\right]\right)=$ $E_{j}$ for all $j, 1 \leq j \leq n$. ([A] denotes the span of $A$.)

- $\left\|P_{n}\right\|=K_{n} \leq c \chi_{u}\left(Z_{2}^{n}\right)$. To justify this, one first shows (see [FJT]) that for any Banach space $E$,

$$
\chi_{u}(E)=\inf \|P\|_{L \rightarrow E},
$$

where the infimum is taken over all Banach lattices $L$ such that $E$ is isometrically embedded in $L$ and all projections $P: L \rightarrow E$. Then observing that $E=Z_{2}^{n}$ have bounded cotype- $q$ constants for a fixed $q<\infty$, we use $[\mathrm{R}$ to reduce to the case when moreover $L$ is supposed to have the same bound on its cotype- $q$ constant. Then, we follow the lines of Proposition 2 of [JLS], using Rademacher embedding.

We shall show that $K_{n} \geq c \log n$, thus proving (2) because it is clear that $K_{n} \leq$ $d\left(Z_{2}^{n}, \ell_{2}^{2 n}\right) \sim \log n$.

Notation. Let $T: Z_{2}^{n} \rightarrow Z_{2}^{n}$ be a linear operator that satisfies $T\left(E_{j}\right) \subset E_{j}$ for $1 \leq j \leq n$. We say that $T$ splits through $\left\{E_{j}\right\}$ or, for short, that $T$ splits. If $T$ splits, the matrices representing $\left.T\right|_{E_{j}}$ in the basis $\left\{e_{j}, f_{j}\right\}, 1 \leq j \leq n$, will be denoted by

$$
\left(\begin{array}{ll}
\alpha_{j} & \beta_{j} \\
\gamma_{j} & \delta_{j}
\end{array}\right) .
$$

If $a=\left(a_{1}, \ldots, a_{n}\right) \in \mathbb{R}^{n}$, the support of $a$ is $\operatorname{supp}(a):=\left\{j: 1 \leq j \leq n, a_{j} \neq 0\right\}$. If $a, b \in \mathbb{R}^{n}, a=\left(a_{1}, \ldots, a_{n}\right), b=\left(a_{1}, \ldots, b_{n}\right)$, we denote $(a, b)=\sum_{1}^{n}\left(a_{j} e_{j}+b_{j} f_{j}\right) \in$ $Z_{2}^{n}$. We also define a vector $a b \in \mathbb{R}^{n}$ by $a b=\left(a_{1} b_{1}, \ldots, a_{n} b_{n}\right)$.

Lemma 2. For any $T$ that splits we have

$$
\max _{j} \max \left\{\left|\alpha_{j}\right|,\left|\beta_{j}\right|,\left|\gamma_{j}\right|,\left|\delta_{j}\right|\right\} \leq\|T\| .
$$

Proof. If $x=e_{j}, 1 \leq j \leq n$, then $\|T x\|=\left|\gamma_{j}\right|+\left|\alpha_{j}\right| \leq\|T\|\|x\|=\|T\|$. If $x=f_{j}$, one similarly obtains $\left|\delta_{j}\right|+\left|\beta_{j}\right| \leq\|T\|$. 
Lemma 3. With the preceding notation, there exists $C>0$ such that for every $T: Z_{2}^{n} \rightarrow Z_{2}^{n}$ that splits and for all $(a, b) \in \mathbb{R}^{2 n}$, we have

$$
\left(\sum_{j=1}^{n}\left(\delta_{j} a_{j}-\alpha_{j} a_{j}+\gamma_{j} a_{j} \log \frac{\left|a_{j}\right|}{\|a\|_{2}}\right)^{2}\right)^{\frac{1}{2}} \leq C\left(\|T\|\|(a, b)\|_{Z_{2}^{n}}+\left(\max _{j \in \operatorname{supp}(a)}\left|\gamma_{j}\right|\right)\|a\|_{2}\right) .
$$

Proof. It is established in $\mathrm{KP}$ that the function $F: \ell_{2}^{n} \rightarrow \ell_{2}^{n}$ given by

$$
F(b)=\left(b_{1} \log \frac{\left|b_{1}\right|}{\|b\|_{2}}, \ldots, b_{n} \log \frac{\left|b_{n}\right|}{\|b\|_{2}}\right) \text { for } b=\left(b_{1}, \ldots, b_{n}\right) \in \mathbb{R}^{n}
$$

is quasi-linear in the sense that for every $a, b \in \mathbb{R}^{n}$,

$$
\|F(a+b)-F(a)-F(b)\|_{2} \leq C\left(\|a\|_{2}+\|b\|_{2}\right) .
$$

We have

$$
\|(\gamma F(a), \gamma a)\|_{Z_{2}^{n}}=\|\gamma a\|_{2}+\|\gamma F(a)-F(\gamma a)\|_{2},
$$

so that

$$
\|\gamma F(a)-F(\gamma a)\|_{2} \leq\|(\gamma F(a), \gamma a)\|_{Z_{2}^{n}}
$$

Hence

$$
\|(\delta-\alpha) a+\gamma F(a)\|_{2} \leq\|(\delta-\alpha) a+F(\gamma a)\|_{2}+\|(\gamma F(a), \gamma a)\|_{Z_{2}^{n}}
$$

Also, unconditionality of the sum (11) implies for every $\theta=\left(\theta_{1}, \ldots, \theta_{n}\right) \in \mathbb{R}^{n}$,

$$
\|(\theta a, \theta b)\|_{Z_{2}^{n}} \leq C \max _{j}\left|\theta_{j}\right|\|(a, b)\|_{Z_{2}^{n}} .
$$

Defining $\alpha=\left(\alpha_{1}, \ldots, \alpha_{n}\right) \in \mathbb{R}^{b}, \beta, \gamma$ and $\delta$ related to $T$ as above, one has

$$
\begin{gathered}
\|(a, b)\|_{Z_{2}^{n}}=\|b\|_{2}+\|a-F(b)\|_{2}, \\
\|T(a, b)\|_{Z_{2}^{n}}=\|\gamma a+\delta b\|_{2}+\|\alpha a+\beta b-F(\gamma a+\delta b)\|_{2}
\end{gathered}
$$

and

$$
\|F(\gamma a+\delta b)-\alpha a\|_{2} \leq 2\|T\|\|(a, b)\|_{Z_{2}^{n}}
$$

because

$$
\begin{gathered}
\|F(\gamma a+\delta b)-\alpha a\|_{2} \leq\|\beta b\|_{2}+\|\alpha a+\beta b-F(\gamma a+\delta b)\|_{2}+\|\gamma a+\delta b\|_{2} \\
\leq\|T(a, b)\|_{Z_{2}^{n}}+\max _{j}\left|\beta_{j}\right|\|b\|_{2} \leq 2\|T\|\|(a, b)\|_{Z_{2}^{n}} .
\end{gathered}
$$

Applying Lemma 2, (3), (5) , (6) and the fact that $\|(F(a), a)\|_{Z_{2}^{n}}=\|a\|_{2}$, we get from (4)

$$
\begin{gathered}
\|(\delta-\alpha) a+\gamma F(a)\|_{2} \leq\|(\delta-\alpha) a+F(\gamma a)\|_{2}+\|(\gamma F(a), \gamma a)\|_{Z_{2}^{n}} \\
\leq\|F(\gamma a)+F(\delta b)-F(\gamma a+\delta b)\|_{2}+\|F(\gamma a+\delta b)-\alpha a\|_{2}+\|\delta a-F(\delta b)\|_{2} \\
+\left(\max _{j \in \operatorname{supp}(a)} \gamma_{j}\right)\|(F(a), a)\|_{Z_{2}^{b}} \\
\leq C\left(\|\gamma a\|_{2}+\|\delta b\|_{2}\right)+2\|T\|\|(a, b)\|_{Z_{2}^{n}}+2\|T\|\|(a, b)\|_{Z_{2}^{n}}+\max _{j \in \operatorname{supp}(a)}\left|\gamma_{j}\right|\|a\|_{2} \\
\leq C\left(\|T\|\|(a, b)\|_{Z_{2}^{n}}+\max _{j \in \operatorname{supp}(a)}\left|\gamma_{j}\right|\|a\|_{2}\right) .
\end{gathered}
$$


Lemma 4. In the context of the preceding lemmas, for every subset $A \subset\{1, \ldots, n\}$, $|A|=k>1$, one has

$$
\left(\frac{1}{k} \sum_{j \in A} \gamma_{j}^{2}\right)^{\frac{1}{2}} \leq \frac{4\|T\|}{\log k}
$$

Proof. Let $x=(a, b) \in Z_{2}^{n}$, where $a=\left(a_{1}, \ldots, a_{n}\right)$ and $b=\left(b_{1}, \ldots, b_{n}\right)$ are given by

$$
a_{j}=\frac{\log \frac{1}{\sqrt{k}}}{\sqrt{k}}, b_{j}=\frac{1}{\sqrt{k}} \text { if } j \in A, a_{j}=b_{j}=0 \text { otherwise }
$$

Then $\|x\|_{Z_{2}^{n}}=1$ and therefore

$$
\|T\| \geq\|T x\|_{Z_{2}^{n}} \geq\left(\frac{1}{k} \sum_{j \in A}\left(-\gamma_{j} \log \sqrt{k}+\delta_{j}\right)^{2}\right)^{\frac{1}{2}} \geq \log \sqrt{k}\left(\frac{1}{k} \sum_{j \in A} \gamma_{j}^{2}\right)^{\frac{1}{2}}-\max _{j}\left|\delta_{j}\right| .
$$

We now use Lemma 2 to complete the proof.

Corollary 5. In the context of the preceding lemmas, let $A \subset\{1, \ldots, n\},|A|=$ $k>1$. Then

a) There exists a subset $A^{\prime} \subset A$, with $\left|A^{\prime}\right| \geq \frac{k}{2}$, such that

$$
\max _{j \in A^{\prime}}\left|\gamma_{j}\right| \leq \frac{4 \sqrt{2}\|T\|}{\log k}
$$

b) If $\operatorname{supp}(a) \subset A^{\prime}$, where $A^{\prime} \subset\{1, \ldots, n\},\left|A^{\prime}\right| \leq k$ and (7) is satisfied, then

$$
\left(\sum_{j=1}^{n}\left(\delta_{j} a_{j}-\alpha_{j} a_{j}+\gamma_{j} a_{j} \log \frac{\left|a_{j}\right|}{\|a\|_{2}}\right)^{2}\right)^{\frac{1}{2}} \leq C\|T\|\|(a, b)\|_{Z_{2}^{n}} .
$$

Proof. Let $C=\left\{j \in A ;\left|\gamma_{j}\right|>\frac{4 \sqrt{2}\|T\|}{\log k}\right\}$. To prove a), it is enough to show that $|C| \leq k / 2$. If it was not true, one would have

$$
\left(\frac{1}{k} \sum_{j \in A} \gamma_{j}^{2}\right)^{\frac{1}{2}} \geq\left(\frac{1}{k} \sum_{j \in C} \gamma_{j}^{2}\right)^{\frac{1}{2}}>\frac{4\|T\|}{\log k}
$$

which contradicts Lemma 4. Part b) follows from Lemma 3 and the fact (see $\underline{\mathrm{KP}}$ ) that the norm of the operator $p_{k}: Z_{2}^{k} \rightarrow \ell_{2}^{k}, p_{k}(a, b)=a$ is equivalent to $\log k$.

Lemma 6. Let $A \subset\{1, \ldots, n\},|A|=k>k_{0}>1$. Then there exists a subset $A^{\prime \prime} \subset A$, with $\left|A^{\prime \prime}\right| \geq \frac{\sqrt{k}}{3}$, such that for all $j \in A^{\prime \prime}$ we have

$$
\left|\gamma_{j}\right| \leq \frac{C\|T\|}{(\log k)^{2}} \quad \text { and } \quad\left|\delta_{j}-\alpha_{j}\right| \leq \frac{C\|T\|}{\log k}
$$

Proof. Let $A^{\prime} \subset A$ with $k^{\prime}=\left|A^{\prime}\right| \geq \frac{k}{2}$ satisfy (7). Let $x=(a, b) \in Z_{2}^{n}$ be defined as in the proof of Lemma 4 , with $A^{\prime}$ replacing $A$. Then, by Corollary $5 \mathrm{~b}$ ), we have (since $\|x\|=1$ )

$$
\left(\frac{1}{k^{\prime}} \sum_{j \in A^{\prime}}\left(\delta_{j}-\alpha_{j}-\gamma_{j} \log \sqrt{k^{\prime}}\right)^{2}\right)^{\frac{1}{2}} \leq \frac{C\|T\|}{\log \sqrt{k^{\prime}}}
$$


As in the proof of Corollary [5, there exists a subset $B \subset A^{\prime}$ with $k^{\prime \prime}=|B| \geq \frac{k^{\prime}}{2} \geq \frac{k}{4}$ such that

$$
\left|\delta_{j}-\alpha_{j}-\gamma_{j} \log \sqrt{k^{\prime}}\right| \leq \frac{\sqrt{2} C\|T\|}{\log \sqrt{k^{\prime}}}=\frac{2 C_{1}\|T\|}{\log k^{\prime}} \text { for all } j \in B .
$$

Now take any subset $B^{\prime} \subset B$ with $\left|B^{\prime}\right|=k^{\prime \prime \prime}=\sqrt{k^{\prime}} \leq \frac{k^{\prime}}{2} \leq k^{\prime \prime}$ and a new vector $x \in Z_{2}^{n}$ like the one above, with $B^{\prime}$ replacing $A^{\prime}$ (the implicit assumption that $\sqrt{k^{\prime}}$ is an integer can easily be dealt with). The same argument as above provides a subset $A^{\prime \prime} \subset B^{\prime}$ with

$$
\left|A^{\prime \prime}\right| \geq \frac{k^{\prime \prime \prime}}{2}=\frac{\sqrt{k^{\prime}}}{2} \geq \frac{\sqrt{k}}{2 \sqrt{2}}
$$

such that

$$
\left|\delta_{j}-\alpha_{j}-\gamma_{j} \log \sqrt{k^{\prime \prime \prime}}\right| \leq \frac{\sqrt{2} C\|T\|}{\log \sqrt{k^{\prime \prime \prime}}}=\frac{4 C_{1}\|T\|}{\log k^{\prime}} \text { for all } j \in A^{\prime \prime} .
$$

Adding (86) and (9) together we get for $j \in A^{\prime \prime}$ :

$$
\begin{gathered}
\frac{\left|\gamma_{j}\right| \log k^{\prime}}{4}=\left|\frac{1}{2} \gamma_{j} \log k^{\prime}-\frac{1}{4} \gamma_{j} \log k^{\prime}\right| \\
=\left|\left(-\delta_{j}+\alpha_{j}+\gamma_{j} \log \sqrt{k^{\prime}}\right)+\left(\delta_{j}-\alpha_{j}-\gamma_{j} \log \sqrt{k^{\prime \prime \prime}}\right)\right| \leq \frac{6 C_{1}\|T\|}{\log k^{\prime}},
\end{gathered}
$$

and thus

$$
\left|\gamma_{j}\right| \leq \frac{24 C_{1}\|T\|}{\left(\log k^{\prime}\right)^{2}} \leq \frac{C_{2}\|T\|}{(\log k)^{2}} .
$$

From (9) and (10) together we get

$$
\left|\delta_{j}-\alpha_{j}\right| \leq \frac{4 C_{1}\|T\|}{\log k^{\prime}}+\left|\gamma_{j}\right| \log \sqrt{k^{\prime \prime \prime}} \leq \frac{4 C_{1}\|T\|}{\log k^{\prime}}+\frac{24 C_{1}\|T\|}{\left(\log k^{\prime}\right)^{2}} \cdot \frac{1}{4} \log k^{\prime} \leq \frac{C_{3}\|T\|}{\log k} \text { for } j \in A^{\prime \prime} .
$$

Proof of Theorem 1. We now recall the construction from [JLS]: Under the hypotheses that $Z_{2}$ has l.u.st., they constructed an operator $T: Z_{2} \rightarrow Z_{2}$ that provided a counterexample to that hypotheses. In the context of $Z_{2}^{n}$, this construction, instead of providing a counterexample, will provide a lower bound estimate of $\chi_{u}\left(Z_{2}^{n}\right)$.

Let the Banach space $Y_{n}$ with the unconditional basis $\left\{y_{j, i} ; j=1, \ldots, n, i=\right.$ $\left.1, \ldots, k_{j}\right\}$ and the projection $P_{n}: Y_{n} \rightarrow Z_{2}^{n}$ be those presented at the opening. For a fixed $1 \leq j \leq n$ a set $A_{j} \subset\left\{1, \ldots, k_{j}\right\}$ is selected and an operator $T_{A_{j}}$ : $\left[e_{j}, f_{j}\right] \rightarrow\left[e_{j}, f_{j}\right]$ is defined by

$$
T_{A_{j}}(x)=P_{n}\left(\sum_{i \in A_{j}} y_{j, i}^{*}(x) y_{j, i}\right),
$$

where $\left\{y_{j, i}^{*}\right\} \subset Y_{n}^{*}$ are the bi-orthogonal functionals of $\left\{y_{j, i}\right\}$. Thus $T_{A_{j}}=\sum_{i \in A_{j}} T_{j, i}$, where $T_{j, i}$ is the rank- 1 operator $T_{j, i}(x)=P_{n}\left(y_{j, i}^{*}(x) y_{j, i}\right)$. We may assume that $T_{j, i} \neq 0$, as otherwise $y_{j, i}$ can be dropped from the basis. Being a rank-1 operator, the matrix representing $T_{j, i}$ is of the form

$$
\left(\begin{array}{ll}
a_{i} & b_{i} \\
\theta_{i} a_{i} & \theta_{i} b_{i}
\end{array}\right) \text { (case I) or }\left(\begin{array}{ll}
0 & 0 \\
a_{i} & b_{i}
\end{array}\right) \text { (case II) }
$$


(note that we dropped the index $j$ for simplicity). In case II we define $\theta_{i}$ to be 1 . We have $\sum_{i=1}^{k_{j}} \theta_{i} b_{i}=1$ (because $\sum_{i} T_{j, i} f_{j}=\left(\sum_{i} \theta_{i} b_{i}\right) f_{j}=f_{j}$ ) and $\sum_{i=1}^{k_{j}}\left|b_{i}\right| \leq K_{n}$.

Define

We have

$$
B_{j}:=\left\{i \in\left\{1, \ldots, k_{j}\right\} ;\left|\theta_{i}\right| \geq \frac{1}{2 K_{n}}\right\} .
$$

$$
\sum_{i \in B_{j}} \theta_{i} b_{i}>\frac{1}{2}
$$

For each $i \in B_{j}$ at least one of the following four possibilities holds:

(८) $\quad \theta_{i} a_{i}>\frac{1}{4 K_{n}}\left|\theta_{i} b_{i}\right|$,

(८८) $-\theta_{i} a_{i}>\frac{1}{4 K_{n}}\left|\theta_{i} b_{i}\right|$

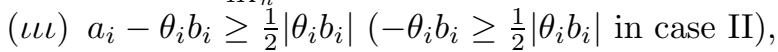

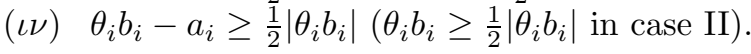

In fact, if $\left|\theta_{i} a_{i}\right|>\frac{1}{4 K_{n}}\left|\theta_{i} b_{i}\right|$, then $(\iota)$ or $(\iota \iota)$ holds. Otherwise $\left|\theta_{i} a_{i}\right| \leq \frac{1}{4 K_{n}}\left|\theta_{i} b_{i}\right|$. Since $i \in B_{j}$ this implies in case I that

$$
\frac{1}{2 K_{n}}\left|a_{i}\right| \leq \frac{1}{4 K_{n}}\left|\theta_{i} b_{i}\right|
$$

which is

$$
\left|\frac{a_{i}}{\theta_{i}}\right| \leq \frac{1}{2}\left|b_{i}\right|
$$

We thus have

$$
\left|\frac{a_{i}}{\theta_{i}}-b_{i}\right| \geq\left|b_{i}\right|-\left|\frac{a_{i}}{\theta_{i}}\right| \geq\left|b_{i}\right|-\frac{1}{2}\left|b_{i}\right|=\frac{1}{2}\left|b_{i}\right|,
$$

which is either $(\iota \iota \iota)$ or $(\iota \nu)$. The remarks about case II are trivial.

It follows that there exists a subset $A_{j} \subset B_{j}$ such that one and the same possibility out of $(\iota)-(\iota \nu)$ holds for all $i \in A_{j}$ and $\sum_{i \in A_{j}} \theta_{i} b_{i}>\frac{1}{8}$. The operator $T: Z_{2}^{n} \rightarrow Z_{2}^{n}$ that splits and is defined by $\left.T\right|_{E_{j}}=T_{A_{j}}$ satisfies $\|T\| \leq K_{n}$. Assume that the matrix representing $T_{A_{j}}$ in the basis $\left\{e_{j}, f_{j}\right\}$ is, as before,

$$
\left(\begin{array}{ll}
\alpha_{j} & \beta_{j} \\
\gamma_{j} & \delta_{j}
\end{array}\right)
$$

From the above it follows that $\delta_{j}>\frac{1}{8}$ for all $j$.

If $(\iota)$ or $(\iota \iota)$ is satisfied for all $i \in A_{j}$, then we have

$$
\left|\gamma_{j}\right|>\frac{1}{4 K_{n}} \delta_{j}>\frac{1}{32 K_{n}} .
$$

If $(\iota \iota \iota)$ or $(\iota \nu)$ is satisfied for all $i \in A_{j}$, then we have

$$
\left|\alpha_{j}-\delta_{j}\right|>\frac{1}{2} \delta_{j}>\frac{1}{16} .
$$

We can now select a subset $D \subset\{1, \ldots, n\}$, with $|D| \geq \frac{n}{2}$, such that either (11) holds for all $j \in D$ or (12) holds for all $j \in D$. We take $D$ to be the set $A$ of Lemma 6. Let $A^{\prime \prime} \subset D$ be as in Lemma 6 and $j \in A^{\prime \prime}$.

If the members of $D$ satisfy (11), then we have

$$
\frac{1}{32 K_{n}} \leq\left|\gamma_{j}\right| \leq \frac{C\|T\|}{\left(\log \frac{n}{2}\right)^{2}} \leq \frac{C_{1} K_{n}}{(\log n)^{2}}
$$


and we conclude

$$
K_{n} \geq c \log n
$$

for some positive constant $c$.

If the members of $D$ satisfy (12), then we have

$$
\frac{1}{16} \leq\left|\delta_{j}-\alpha_{j}\right| \leq \frac{C\|T\|}{\log \frac{n}{2}} \leq \frac{C_{1} K_{n}}{\log n}
$$

thus

$$
K_{n} \geq c \log n
$$

\section{The ASYMmetRY of $Z_{2}^{n}$}

Definition. We say that a subgroup $G$ of $G L_{k}(\mathbb{R})$ is rich if it is a compact group and that every operator in $L\left(\mathbb{R}^{k}\right)$ which commutes with all elements of $G$ is a scalar multiple of the identity on $\mathbb{R}^{k}$. It is well known that for any compact subgroup $G$ of $G L_{k}(\mathbb{R})$, there exists a compact subgroup $H$ of $\mathcal{O}_{k}$ (the orthogonal group) such that $G=\left\{V^{-1} h V ; h \in H\right\}$, where $V \in L\left(\mathbb{R}^{k}\right)$ is some linear invertible operator. If $G$ is a rich subgroup of $G L_{k}(\mathbb{R})$, we define the measure of symmetry $s_{G}(E)$ of a $k$-dimensional normed space $E$ with respect to $G$ by

$$
s_{G}(E):=\int_{G}\|g\|_{E \rightarrow E} d \mu(g),
$$

where $\mu$ is the normalized Haar measure on $G$. We define

$$
s(E)=\inf \left\{s_{G}(E) ; G \text { a rich subgroup }\right\} .
$$

Note that

$$
1 \leq s(E) \leq S(E):=\inf \left\{\sup _{g \in G}\|g\|_{E \rightarrow E} ; G \text { a rich subgroup }\right\} .
$$

The quantity $S(E)$, called the asymmetry constant of $E$, was defined originally in [GG] and further discussed in [GL1]. If $S(E)=1$ we say that $E$ has enough symmetries. This is the case for spaces with 1-symmetric basis (take the rich group of permutations and changes of signs on the basis). In connection with this quantity we have the following partial result:

Theorem 7. For every rich subgroup $G$ of $\mathcal{O}_{2 n}$ one has

$$
s_{G}\left(Z_{2}^{n}\right)=\int_{G}\|g\|_{Z_{2}^{n} \rightarrow Z_{2}^{n}} d \mu(g) \sim \log n \sim \max _{g \in G}\|g\|_{Z_{2}^{n} \rightarrow Z_{2}^{n}},
$$

where $\mu$ denotes the normalized Haar measure on $G$.

We use the following lemma.

Lemma 8. Let a couple $E$ and $F$ of $k$-dimensional normed spaces, an ideal norm $\alpha$ and a rich subgroup $G$ of $G L_{k}(\mathbb{R})$ be given. Then for every invertible operator $S \in L\left(\mathbb{R}^{k}\right)$, one has

$$
k \leq \alpha(S: E \rightarrow F) \alpha^{*}\left(S^{-1}: F \rightarrow E\right) \leq k \int_{G}\left\|S^{-1} g S\right\|_{E \rightarrow E}\left\|g^{-1}\right\|_{F \rightarrow F} d \mu(g),
$$


where $\alpha^{*}$ denotes the conjugate ideal norm of $\alpha$. In particular, if all elements of $G$ are isometries of $F$, then

$$
k \leq \alpha(S: E \rightarrow F) \alpha^{*}\left(S^{-1}: F \rightarrow E\right) \leq k \int_{G}\left\|S^{-1} g S\right\|_{E \rightarrow E} \quad d \mu(g) .
$$

Proof. We first prove for any $k$-dimensional normed spaces $D$ and $F$ on $\mathbb{R}^{n}$ that if $I: \mathbb{R}^{k} \rightarrow \mathbb{R}^{k}$ denotes the identity operator, then

$$
k \leq \alpha(I: D \rightarrow F) \alpha^{*}\left(I^{-1}: F \rightarrow D\right) \leq k \int_{G}\|g\|_{D \rightarrow D}\left\|g^{-1}\right\|_{F \rightarrow F} d \mu(g) .
$$

By the definition of $\alpha^{*}$, there exists $U \in L\left(\mathbb{R}^{k}\right)$ such that

$$
\alpha(U: D \rightarrow F) \alpha^{*}\left(I^{-1}: F \rightarrow D\right)=\operatorname{trace}\left(U I^{-1}\right)=\operatorname{trace}(U) .
$$

Let $V=\int_{G} g^{-1} U g d \mu(g)$ be considered as an operator from $D$ to $F$. Since $G$ is a rich subgroup, one has $V=c I$ for some $c \in \mathbb{R}$, so that $\operatorname{trace}(U)=\operatorname{trace}(V)=k c$. One clearly has

$$
\begin{gathered}
c \alpha(I: D \rightarrow F)=\alpha(V: D \rightarrow F) \leq \int_{G} \alpha\left(g^{-1} U g: D \rightarrow F\right) d \mu(g) \\
\leq \alpha(U: D \rightarrow F) \int_{G}\left\|g^{-1}\right\|_{F \rightarrow F}\|g\|_{D \rightarrow D} d \mu(g) .
\end{gathered}
$$

It follows that

$$
\begin{gathered}
\alpha(I: D \rightarrow F) \alpha^{*}\left(I^{-1}: F \rightarrow D\right) \\
\leq \frac{\alpha(U: D \rightarrow F) \alpha^{*}\left(I^{-1}: F \rightarrow D\right)}{c} \int_{G}\|g\|_{D \rightarrow D}\left\|g^{-1}\right\|_{F \rightarrow F} d \mu(g) \\
=k \int_{G}\|g\|_{D \rightarrow D}\left\|g^{-1}\right\|_{F \rightarrow F} d \mu(g) .
\end{gathered}
$$

This proves (18). We define a normed space $D$ by setting $\|x\|_{D}=\left\|S^{-1} x\right\|_{E}$. Then $S^{-1}: D \rightarrow E$ and $S: E \rightarrow D$ are isometries, and one has

$$
\begin{gathered}
\alpha(I: D \rightarrow F)=\alpha(S: E \rightarrow F), \alpha^{*}\left(I^{-1}: F \rightarrow D\right)=\alpha^{*}\left(S^{-1}: F \rightarrow E\right) \\
\text { and }\|g\|_{D \rightarrow D}=\left\|S^{-1} g S\right\|_{E \rightarrow E} .
\end{gathered}
$$

Together with (18) this implies (16) .

Proof of Theorem 17. We shall use Lemma 8 with $S=I: Z_{2}^{n} \rightarrow \ell_{2}^{2 n}$, where $I$ is the identity sending the basis $e_{j}, f_{j} 1 \leq j \leq n$ on the canonical basis of $\ell_{2}^{2 n}$. Here we present two proofs: $\mathbf{A}$ and $\mathbf{B}$.

A. Let $\alpha=\Pi_{1}$ and $\alpha^{*}=\gamma_{\infty}$, the ideal norm of factorization through $L_{\infty}$.

a. We claim that

$$
\Pi_{1}\left(I: Z_{2}^{n} \rightarrow \ell_{2}^{2 n}\right) \geq c \sqrt{n} \log n .
$$

In fact, define, for $1 \leq j \leq n, x_{j} \in Z_{2}^{n}$ by

$$
x_{j}=\frac{1}{\sqrt{n}}\left(\log \frac{1}{\sqrt{n}}\right) e_{j}+\frac{1}{\sqrt{n}} f_{j} .
$$


Then $\left\|I\left(x_{j}\right)\right\|_{2} \sim \frac{\log n}{\sqrt{n}}$, and for every choice of signs $\varepsilon=\left(\varepsilon_{1}, \ldots, \varepsilon_{n}\right) \in\{-1,1\}^{n}$, one has

$$
\left\|\sum_{j=1}^{n} \varepsilon_{j} x_{j}\right\|_{Z_{2}^{n}}=1
$$

Hence

$$
\Pi_{1}\left(I: Z_{2}^{n} \rightarrow \ell_{2}^{2 n}\right) \geq \frac{\sum_{j=1}^{n}\left\|x_{j}\right\|_{2}}{\sup _{\varepsilon}\left\|\sum_{j=1}^{n} \varepsilon_{j} x_{j}\right\|_{Z_{2}^{n}}} \geq c \sqrt{n} \log n .
$$

b. Define $P: Z_{2}^{n} \rightarrow \ell_{2}^{n}$ by $P(a, b)=b$. Then $\|P\|_{Z_{2}^{n} \rightarrow \ell_{2}^{n}}=1$, and if $W: \ell_{2}^{n} \rightarrow \ell_{2}^{2 n}$ is the embedding defined by $W(b)=(0, b)$, then

$$
\begin{gathered}
\gamma_{\infty}\left(I^{-1}: \ell_{2}^{2 n} \rightarrow Z_{2}^{n}\right)=\|P\|_{Z_{2}^{n} \rightarrow \ell_{2}^{n}} \gamma_{\infty}\left(I^{-1}: \ell_{2}^{2 n} \rightarrow Z_{2}^{n}\right)\|W\|_{\ell_{2}^{n} \rightarrow \ell_{2}^{2 n}} \\
\geq \gamma_{\infty}\left(P I^{-1} W: \ell_{2}^{n} \rightarrow \ell_{2}^{n}\right) \sim \sqrt{n},
\end{gathered}
$$

because $P I^{-1} W: \ell_{2}^{n} \rightarrow \ell_{2}^{n}$ is actually the identity mapping on $\ell_{2}^{n}$.

c. It follows that

$$
\Pi_{1}\left(I: Z_{2}^{n} \rightarrow \ell_{2}^{2 n}\right) \gamma_{\infty}\left(I^{-1}: \ell_{2}^{2 n} \rightarrow Z_{2}^{n}\right) \geq c n \log n .
$$

B. Let $\alpha$ be the operator norm; then $\alpha^{*}=i_{1}$, the integral norm. It is easy to see that

$$
\|I\|_{Z_{2}^{n} \rightarrow \ell_{2}^{2 n}} \sim \log n
$$

and

$$
\begin{aligned}
& i_{1}\left(I^{-1}: \ell_{2}^{2 n} \rightarrow Z_{2}^{n}\right)=i_{1}\left(I^{-1}: \ell_{2}^{2 n} \rightarrow Z_{2}^{n}\right)\|P\|_{Z_{2}^{n} \rightarrow \ell_{2}^{n}} \\
& \geq i_{1}\left(P I^{-1}: \ell_{2}^{2 n} \rightarrow \ell_{2}^{n}\right)=\sup _{S \neq 0} \frac{\operatorname{trace}\left(P I^{-1} S\right)}{\|S\|_{\ell_{2}^{n} \rightarrow \ell_{2}^{2 n}}} \geq n .
\end{aligned}
$$

It follows that

$$
\|I\|_{Z_{2}^{n} \rightarrow \ell_{2}^{2 n}} \quad i_{1}\left(I^{-1}: \ell_{2}^{2 n} \rightarrow Z_{2}^{n}\right) \geq c n \log n .
$$

Using either $\mathbf{A}$ or $\mathbf{B}$, together with Lemma 8 with $F=\ell_{2}^{2 n}, E=Z_{2}^{n}$ and $S=I$, we get that

$$
\int_{G}\|g\|_{Z_{2}^{n} \rightarrow Z_{2}^{n}} d \mu(g) \geq c \log n
$$

It follows that

$$
\log n \leq \frac{1}{c} \int_{G}\|g\|_{Z_{2}^{n} \rightarrow Z_{2}^{n}} d \mu(g) \leq \frac{1}{c} \max _{g \in G}\|g\|_{Z_{2}^{n} \rightarrow Z_{2}^{n}} \leq d\left(Z_{2}^{n}, \ell_{2}^{2 n}\right) \sim \log n,
$$

where here $d$ denotes the Banach-Mazur distance. This proves (15).

Remark. One can prove in the same way as A.a. in the proof of the last theorem that if $I: Z_{2}^{n} \rightarrow \ell_{\infty}^{2 n}$ is the identity mapping sending the basis $\left(e_{j}, f_{j}\right), 1 \leq j \leq n$, on the canonical basis of $\ell_{\infty}^{2 n}$, then

$$
\Pi_{1}\left(I: Z_{2}^{n} \rightarrow \ell_{\infty}^{2 n}\right) \geq c \sqrt{n} \log n .
$$

We also have that

$$
\gamma_{\infty}\left(I^{-1}: \ell_{\infty}^{2 n} \rightarrow Z_{2}^{n}\right)=\left\|I^{-1}\right\|_{\ell_{\infty}^{2 n} \rightarrow Z_{2}^{n}} \sim \sqrt{n} \log n .
$$


It follows from Lemma 8 that for every rich subgroup $G$ of $G L_{2 n}(\mathbb{R})$, one has

$$
\begin{gathered}
c n(\log n)^{2} \leq \Pi_{1}\left(I: Z_{2}^{n} \rightarrow \ell_{\infty}^{2 n}\right) \gamma_{\infty}\left(I^{-1}: \ell_{\infty}^{2 n} \rightarrow Z_{2}^{n}\right) \\
\leq n \int_{G}\left\|g^{-1}\right\|_{\ell_{\infty}^{2 n} \rightarrow \ell_{\infty}^{2 n}}\|g\|_{Z_{2}^{n} \rightarrow Z_{2}^{n}} d \mu(g) .
\end{gathered}
$$

The first inequality is actually an equivalence, in view of the fact that if one takes for $G$ the group of changes of signs and permutaions of indices of the basis, one has $\left\|g^{-1}\right\|_{\ell_{\infty}^{2 n} \rightarrow \ell_{\infty}^{2 n}}=1$ for all $g \in G$ and

$$
\int_{G}\|g\|_{Z_{2}^{n} \rightarrow Z_{2}^{n}} d \mu(g) \leq\left\|I: Z_{2}^{n} \rightarrow \ell_{\infty}^{2 n}\right\|\left\|I^{-1}: \ell_{\infty}^{2 n} \rightarrow Z_{2}^{n}\right\| \leq c(\log n)^{2}
$$

as $\left\|I: Z_{2}^{n} \rightarrow \ell_{\infty}^{2 n}\right\| \sim \log n$.

Added in proof. After the paper was processed, we realized that answers to some of the questions posed at the end of the introduction had already been given in the paper $[\mathrm{KT}$. In particular, examples of $n$-dimensional Banach spaces with bounded GL-constant and l.u.st constant greater than $\mathrm{cn}^{\alpha}$ (with $\alpha$ as large as 1/6) are given there.

\section{REFERENCES}

[CK] Casazza P. G. and Kalton N. J.: Unconditional bases and unconditional finite-dimensional decompositions in Banach spaces. Israel J. Math. 95 (1996), 349-373. MR.1418300 (97k:46010)

[FJT] Figiel T., Johnson W.B. and Tzafriri L.: On Banach lattices and spaces having local unconditional structure, with applications to Lorentz function spaces. Collection of articles dedicated to G. G. Lorentz on the occasion of his sixty-fifth birthday, IV. J. Approximation Theory 13 (1975), 395-412. MR0367624(51:3866)

[GG] Garling D. J. H. and Gordon Y.: Relations between some constants associated with finite dimensional Banach spaces. Israel J. Math. 9 (1971), 346-361. MR0412775 (54:896)

[GL1] Gordon Y. and Lewis D. R.: Absolutely summing operators and local unconditional structures. Acta Math. 133 (1974), 27-48. MR0410341 (53:14091)

[GL2] Gordon Y. and Lewis D. R.: Banach ideals on Hilbert spaces. Studia Math. 54 (1975), no. 2, 161-172. MR0388149 (52:8986)

[JLS] Johnson W. B., Lindenstrauss J. and Schechtman G.: On the relation between several notions of unconditional structure. Israel J. Math. 37 (1980), 120-129. MR599307 (83f:46015)

[KP] Kalton N. J. and Peck N. T.: Twisted sums of sequence spaces and the three space problem. Trans. Amer. Math. Soc. 255 (1979), 1-30. MR542869 (82g:46021)

$[\mathrm{KT}]$ Komorowski R.A. and Tomczak-Jaegermann N.: Subspaces of $l_{2}(X)$ and $\operatorname{Rad}(X)$ without local unconditional structure. Studia Math. 149 (2002), no. 1, 1-21. MR.1881713 (2003c:46016)

[LT1] Lindenstrauss J. and Tzafriri L.: Classical Banach spaces. I. Springer-Verlag, BerlinHeidelberg-New York, 1977. MR0500056 (58:17766)

[LT2] Lindenstrauss J. and Tzafriri L.: Classical Banach spaces. II. Springer-Verlag, BerlinHeidelberg-New York, 1979. MR.540367 (81c:46001)

[PW] Pelczynski A. and Wojciechowski M.: Spaces of functions with bounded variation and Sobolev spaces without local unconditional structure. J. Reine Angew. Math. 558 (2003), 109-157. MR1979184(2004c:46058)

[P] Pietsch A.: Operator Ideals. VEB Deutsch. Ver. Wiss., Berlin, 1978; and North-Holland Publ. Co., Amsterdam-New York, 1980. MR582655 (81j:47001) 
[R] Reisner S.: Operators which factor through convex Banach lattices. Canad. J. Math. 32 (1980), no. 6, 1482-1500. MR604702 (82k:47031)

[T] Tomczak-Jaegermann N.: Banach-Mazur distances and finite-dimensional operator ideals. Pitman Monographs and Surveys in Pure and Applied Mathematics, 38. Longman Scientific and Technical, Harlow; copublished in the United States with John Wiley and Sons, Inc., New York, 1989. MR.993774 (90k:46039)

Department of Mathematics, Technion, Israel Institute of Technology, Haifa 32000, ISRAEL

E-mail address: gordon@techunix.technion.ac.il

Department of Mathematics, University of Illinois, Urbana, Illinois 61801

E-mail address: junge@math.uiuc.edu

Laboratoire d'Analyse et de Mathématiques Appliquées (UMR 8050), Université Paris-Est-Marne-la-Vallée, Cité Descartes-5, Bd Descartes, Champs-sur-Marne, 77454 Marne-la-Vallée Cedex 2, France

E-mail address: Mathieu.Meyer@univ-mlv.fr

Department of Mathematics, University of Haifa, Haifa 31905, Israel

E-mail address: reisner@math.haifa.ac.il 